\title{
ELICITING HARMONICS ON STRINGS
}

\author{
Steven J. Cox ${ }^{1}$ And Antoine Henrot ${ }^{2}$
}

\begin{abstract}
One may produce the $q$ th harmonic of a string of length $\pi$ by applying the 'correct touch' at the node $\pi / q$ during a simultaneous pluck or bow. This notion was made precise by a model of Bamberger, Rauch and Taylor. Their 'touch' is a damper of magnitude $b$ concentrated at $\pi / q$. The 'correct touch' is that $b$ for which the modes, that do not vanish at $\pi / q$, are maximally damped. We here examine the associated spectral problem. We find the spectrum to be periodic and determined by a polynomial of degree $q-1$. We establish lower and upper bounds on the spectral abscissa and show that the set of associated root vectors constitutes a Riesz basis and so identify 'correct touch' with the $b$ that minimizes the spectral abscissa.
\end{abstract}

Mathematics Subject Classification. 35P10, 35P15, 74K05, 74P10.

Received September 5, 2006.

Published online January 18, 2008.

\section{INTRODUCTION}

We are interested in one's capacity to elicit harmonic overtones, or simply 'harmonics', from stringed instruments. Such questions have long vexed theorists, though not practitioners, and were key points of friction in the development of mechanics and analysis in the period between Newton and Cauchy. Although Truesdell [30], Cannon and Dostrovsky [6] and Christensen [7] cover this period in fascinating detail, their focus on the intellectual developments of D'Alembert, Bernoulli, Euler, Lagrange and Rameau, leaves a number of practical innovations, notably harmonics, in the dark. As the associated mathematical question was in fact well set by 1692 and yet its mathematical analysis did not commence until 1982 we open with an outline of the full history.

Though overtones, or upper partials, had been heard through the ages they were not seen until late in the 17th century. More precisely, though Descartes, Mersenne and Galileo each remark on the sound of overtones and often ascribe them to sympathetic vibrations with neighboring bodies, Wallis [32] in 1677 is the first to report on the means by which one may see the nodes of a vibrating string. He uses one string to excite a higher mode in a second, on which are suspended paper riders, and notes that, when the first is tuned an octave above the second, and the first is struck, "the two halves of this other [second] will both tremble; but not the middle point". He proceeds to find the nodes of what we call the third and fourth modes by tuning the first string a twelfth and double octave, respectively, above the second and observes that these nodes divide the string in equal, or aliquot, parts. Roberts [27] in 1692 was the first to observe that, by softly pressing at a node while bowing elsewhere, higher modes could be elicited on a single string. He developed these ideas in his explication of the trumpet marine, or nun's fiddle, a curious variant of the ancient monochord on which the only pleasant

\footnotetext{
Keywords and phrases. Point-wise damping, spectral abscissa, Riesz basis.

1 Computational and Applied Mathematics, Rice University, Houston, TX, USA; cox@caam.rice.edu

2 Institut Élie Cartan, UMR 7502, Nancy Université - CNRS - INRIA, Nancy, France.
} 
tones are harmonics. Roberts writes "Now in the Trumpet-Marine you do not stop close as in other instruments, but touch the String gently with your thumb, whereby there is mutual concurrence of the upper and lower part of the String to produce the sound" and observes "that the Trumpet Marine will yield no musical sound but when the stop makes the upper part of the string an aliquot of the remainder, and consequently of the whole". Roberts concludes with a lovely figure that includes both a clear indication of the first three theoretical mode shapes and a practical diagram of the 15 nodes at which the player ought to gently thumb.

After Roberts the theory and practice of harmonics regrettably diverge over the course of the 18th century. On the practical side we have Mondonville's opus 4, Les Sons Harmoniques [3], of 1735, the first pieces scored for violin harmonics, complete with a beautiful diagram identifying notes with nodes. On the theoretical side, the mode shapes of Roberts were not to reappear until the 1747 study by Bernoulli [4], a work that stemmed not from Roberts' practical considerations but from the theoretical work of Taylor [29]. In struggling to understand how a single string could simultaneously support so many modes, Bernoulli, Euler and Lagrange, see [6,23,26], brought forth trigonometric series but rejected these in favor of Euler and D'Alembert's general wave solution and vague reference to sympathetic resonance. None of these four men were aware of the simple mechanical means, of Roberts and Mondonville, for eliciting these individual modes. This is remarkable given the presence of Rameau, a composer and music theorist, hailed as the 'Newton of Harmony', (see [6], p. 8), who not only entered into substantive individual correspondence with Bernoulli, Euler, Lagrange and D'Alembert but who was also surely aware of both the strange flageolet tones of the trumpet marine and the works of Mondonville. Regarding Rameau's influence on Bernoulli et al. see Truesdell [30] and especially Christensen [7]. Galpin [12] informs us that Rameau's tenure with the orchestra of the Académie du Concert of Lyon overlapped with that of Prin, the established master of the trumpet marine. Rameau, in the preface to his Pièces de Clavecin en concert, 1741, speaks of the recent success of such ensemble pieces (most likely Mondonville's opus 5, see Girdlestone [13], p. 43) and names one of his own pieces after Boucon, his student and the wife of Mondonville. And yet, although Rameau [25], p. 8, was aware of the work of Sauveur [28] on overtones, or "petits sons", neither Prin's nor Mondonville's harmonics figure anywhere in Rameau's vast theoretical writings. Rameau's failure to recognize the theoretical importance of their work contributed to a neglect, over the ensuing 100 years, of the study of harmonics. This is, unfortunately, in accord with Miller's dark verdict, [24], p. 37, that "A careful search fails to reveal any major contribution to the science of sound which arose in the 18th century".

It is not until 1807 in the work of Young [35], lect. 32, that we find a lucid synthesis of Roberts and Mondonville: "if a long chord be initially divided into any number of such equal portions, its parts will continue to vibrate in the same manner as if they were separate chords; the points of division only remaining always at rest. Such subordinate sounds are called harmonics: they are often produced in violins by lightly touching one of the points of division with the finger, when the bow is applied, and in all such cases it may be shown, by putting small feathers or pieces of paper on the string, that the remaining points of division are also quiescent, while the intervening points are in motion". Although Young's observations were echoed by Helmholtz, 1862, [15], Chapter 4 and illustrated by Tyndall, 1875, [31], Section 3.6, it appears that Rayleigh, 1877, [26], Section 134, in noting that "when but a single point of the string is retarded by friction there are no normal co-ordinates properly so called", was the first to have attempted a mathematical analysis of harmonics. After Rayleigh the problem remained untouched until the beautiful, but little known, 1982 work of Bamberger, Rauch and Taylor [2]. (Coincidentally, the problem of pointwise damping of suspension bridge cables was also first broached by Kovács [18] in 1982.)

Bamberger et al. followed Rayleigh in retarding by friction, $b$, at but a single point, $a$, the motion of a fixed string of length $\pi$ with transverse displacement $u$. More precisely, they considered

$$
u_{t t}-u_{x x}+b \delta(x-a) u_{t}=0, \quad u(0, t)=u(\pi, t)=0,
$$

with initial data in the energy space $X=H_{0}^{1}(0, \pi) \times L^{2}(0, \pi)$ endowed with the inner product

$$
\langle[f, g],[u, v]\rangle=\int_{0}^{\pi}\left\{f^{\prime} \bar{u}^{\prime}+g \bar{v}\right\} \mathrm{d} x .
$$


They showed that if the damping is applied at a rational multiple of $\pi$ then it only damps those modes not vanishing there. They then equated "correct touch" with the value of $b$ that best damps these remaining modes. In the case $a=\pi / 2$ they showed that $b=2$ was the correct touch. We here attack the general case by investigating the spectra of the associated wave operator

$$
\begin{aligned}
A(a, b) & =\left(\begin{array}{cc}
0 & I \\
\partial_{x x} & 0
\end{array}\right), \quad x \neq a \\
D(A(a, b)) & =\left\{[f, g] \in\left(H_{0}^{1}(0, \pi) \cap H^{2}(0, a) \cap H^{2}(a, \pi)\right) \times H_{0}^{1}(0, \pi): f^{\prime}\left(a^{+}\right)-f^{\prime}\left(a^{-}\right)=b g(a)\right\} .
\end{aligned}
$$

Bamberger et al. argue that $A(a, b)$ has a compact resolvent and so discrete spectrum and that when $a=p \pi / q$, where $p$ and $q$ are relatively prime integers, it has the imaginary eigenvalues

$$
\lambda_{1, n}=i n q, \quad n \in \mathbf{Z} \backslash 0
$$

and associated eigenvectors

$$
V_{1, n}=\sin (n q x)[1 /(n q) i]
$$

We write $\sigma(p \pi / q, b)$ for the spectrum of $A(p \pi / q, b)$ and

$$
\sigma_{\mathcal{H}}(p \pi / q, b)=\left\{\lambda_{1, n}\right\}
$$

for the harmonic part of the spectrum. It is shown in [2] that the nonharmonic spectrum, $\sigma(p \pi / q, b) \backslash \sigma_{\mathcal{H}}(p \pi / q, b)$ lies in the strict left half plane and that the semigroup $\mathrm{e}^{t A(p \pi / q, b)}$ enjoys exponential decay on the orthogonal complement of the harmonic subspace

$$
\mathcal{H} \equiv \operatorname{span}\left\{V_{1, n}\right\}
$$

More precisely, denoting the orthogonal complement of $\mathcal{H}$ by $\mathcal{H}^{\perp}$, Bamberger et al. establish the existence of positive constants, $C_{1}$ and $C_{2}$, depending only on $p / q$ and $b$, such that

$$
\left\|\mathrm{e}^{t A(p \pi / q, b)} U\right\|_{X} \leq C_{1} \mathrm{e}^{-C_{2} t}\|U\|_{X} \quad \forall U \in \mathcal{H}^{\perp}, t \geq 0 .
$$

In this work we shall show the greatest $C_{2}$ for which (1.4) holds is the magnitude of the nonharmonic spectral abscissa of $A(p \pi / q, b)$,

$$
\mu(p \pi / q, b) \equiv \max \left\{\Re \lambda: \lambda \in \sigma(p \pi / q, b) \backslash \sigma_{\mathcal{H}}(p \pi / q, b)\right\}
$$

and, along the way, establish bounds on $b \mapsto \mu(a, b)$ and produce explicit minimizers when $a=\pi / 3$ and $a=\pi / 4$. More precisely, in Section 2 we delineate the structure of the spectrum, taking care to identify multiple eigenvalues and to specify the behavior of the spectrum for $b$ near 0,2 and $\infty$. In Section 3 we establish upper and lower bounds on the nonharmonic spectral abscissa and arrive at the correct touch for $a=\pi / 3$ and $a=\pi / 4$. In Section 4 we prove that the associated root vectors comprise a Riesz basis and so identify $\mu$ with the best rate of decay. We close in Section 5 with a return to dimensions and a discussion of the true magnitude of $b$ and the role played by an additional elastic term at $a$.

There exist many works, e.g., Cox and Zuazua [9,10], devoted to the optimal damping of strings, and a sizable mathematical literature, e.g., Liu [22], Jaffard et al. [16], and Ammari et al. [1], and engineering literature, e.g., Kovács [18] and Krenk [21], that in fact focus on pointwise damping. As the focus of these previous efforts was on the optimal damping of either all modes or a single mode, the harmonics problem of Bamberger et al. was naturally neglected.

\section{ON THE Disposition OF THE SPECTRUM}

If $A(a, b)[y z]=\lambda[y z]$ then $z=\lambda y$ and $y$ must satisfy the quadratic eigenvalue problem

$$
y^{\prime \prime}(x)-\lambda^{2} y(x)-\lambda b \delta(x-a) y(x)=0, \quad y(0)=y(\pi)=0 .
$$


It is not difficult to show that

$$
y(x)= \begin{cases}\sinh (\lambda(\pi-a)) \sinh (\lambda x) & \text { if } 0<x<a \\ \sinh (\lambda a) \sinh (\lambda(\pi-x)) & \text { if } a<x<\pi\end{cases}
$$

and $\lambda$ is a zero of the shooting function

$$
S(\lambda ; a, b)=\sinh (\lambda \pi)+b \sinh (\lambda a) \sinh (\lambda(\pi-a)) .
$$

The majority of our results are consequences of the simple observation that

$$
S(\lambda ; p \pi / q, b)=-(1 / 4) \exp (\lambda \pi) P(\exp (-2 \lambda \pi / q) ; b)
$$

where $P$ is the polynomial

$$
P(w ; b)=(2-b) w^{q}+b w^{p}+b w^{q-p}-(2+b) .
$$

In terms of its roots, $\left\{w_{k}=\left|w_{k}\right| \exp \left(i \theta_{k}\right)\right\}_{k=1}^{q}$ (repeated according to their multiplicity), the eigenvalues of $A(a, b)$ are simply

$$
\lambda_{k, n}=\frac{-q}{2 \pi}\left\{\ln \left|w_{k}\right|+i\left(\theta_{k}+2 \pi n\right)\right\}, \quad k=1, \ldots, q, \quad n \in \mathbf{Z} .
$$

For example, if $p / q=1 / 2$ we find $P(w ; b)=(2-b) w^{2}+2 b w-2-b$. Its roots, $w_{1}=1$ and $w_{2}=(b+2) /(b-2)$ translate readily into the associated eigenvalues

$$
\begin{aligned}
& \lambda_{1, n}=i 2 n, \quad n \neq 0 \\
& \lambda_{2, n}=-\frac{1}{\pi} \ln \left|\frac{b+2}{b-2}\right|-i(H(2-b)+2 n)
\end{aligned}
$$

where $H$ is the Heaviside function. It follows that

$$
\mu(\pi / 2, b)=-\frac{1}{\pi} \ln \left|\frac{b+2}{b-2}\right|
$$

approaches $-\infty$ as $b \rightarrow 2$, in agreement with Bamberger et al.

In preparation for the general case we now examine numerical approximation of the spectra for $0<b<\infty$ and $q$ up to 8. Our calculations are facilitated by the fact that the spectrum is completely determined by the polynomial (2.3) and the observation that

$$
S(\bar{\lambda} ; a, b)=\bar{S}(\lambda ; a, b) \quad \text { and } \quad S(\lambda+q i, p \pi / q, b)=(-1)^{q} S(\lambda, p \pi / q, b)
$$

from which we conclude that the spectrum has period $q i$ and that within a period the spectrum is symmetric about $i q / 2$.

In the next 10 panels we have plotted the first $q$ eigenvalues as a function of $b$ as $b$ ascends from 0 to 5 in steps of 0.01 . For $b<2$ we have used left-pointing arrowheads while for $b>2$ we have used right-pointing arrowheads. We have, for greater legibility, limited the plot range real parts in excess of -2 . We have placed a dashed vertical line at each minimal spectral abscissa. Each plot is labeled with its particular value of $a$ and the associated (approximate) minimal spectral abscissa, $\mu_{*}$, and correct touch, $b_{*}$. These approximate values are obtained by solving the discrete minimization problem $\min \{\mu(a, k / 100) ; k=0: 500\}$.

We shall now prove that the root trajectories indeed behave in the simple manner illustrated in Figure 1. Perhaps the first thing that catches one's eye is the crossing of root trajectories when $p$ and $q$ are odd (panels $(\mathrm{D}, \mathrm{F}, \mathrm{H})$ and $(\mathrm{I}))$. We recall that though Bamberger et al. proved that all eigenvalues are geometrically simple they neglected to consider higher algebraic multiplicity. The calculation is explicit when $a=\pi / 3$. For

$$
P(w ; b)=(2-b) w^{3}+b w+b w^{2}-(2+b)=(w-1)\left((2-b) w^{2}+2 w+(2+b)\right)
$$


(A) $a=\pi / 8, \mu_{*}=-0.0791, b_{*}=2.79$

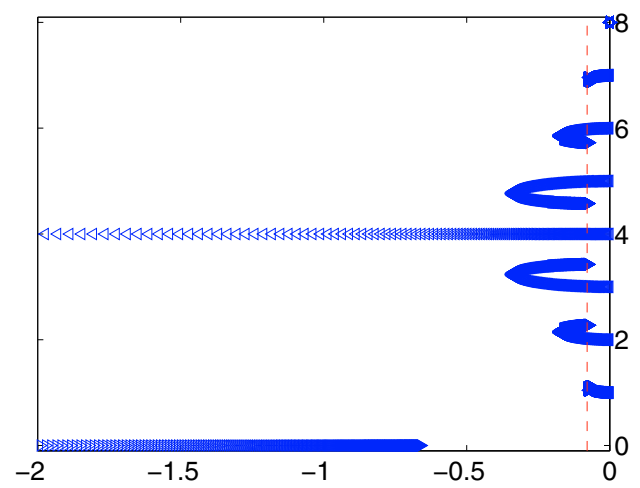

(C) $\mathrm{a}=3 \pi / 8, \mu_{*}=-0.145, \mathrm{~b}_{*}=3.02$

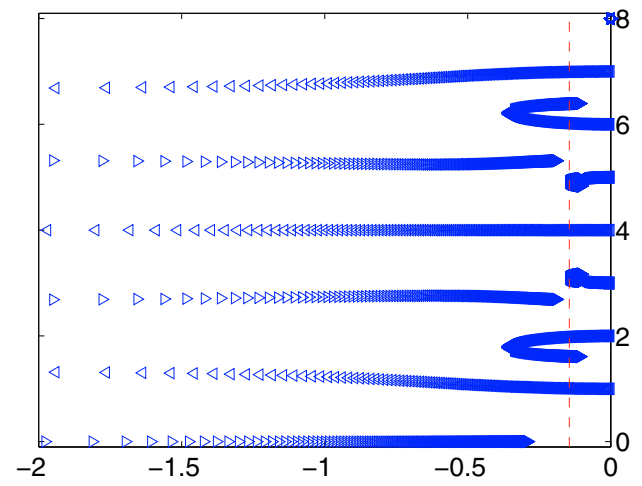

(E) $\quad \mathrm{a}=2 \pi / 7, \mu_{*}=-0.129, \mathrm{~b}_{*}=2.64$

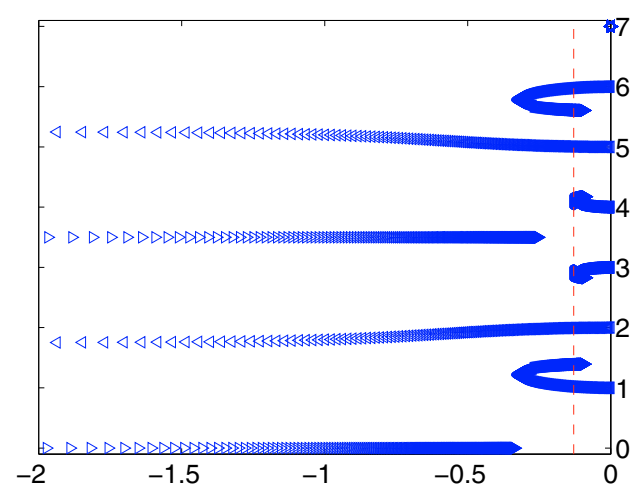

(B) $a=\pi / 4, \mu_{*}=-0.235, b_{*}=1.7$

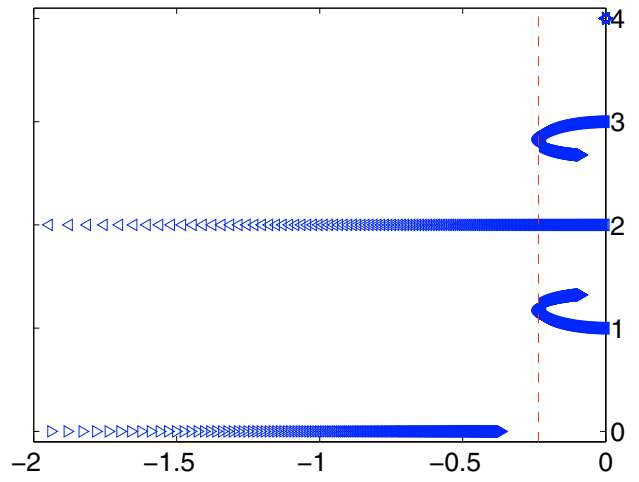

(D) $\quad \mathrm{a}=\pi / 7, \mu_{\star}=-0.0944, \mathrm{~b}_{*}=2.49$

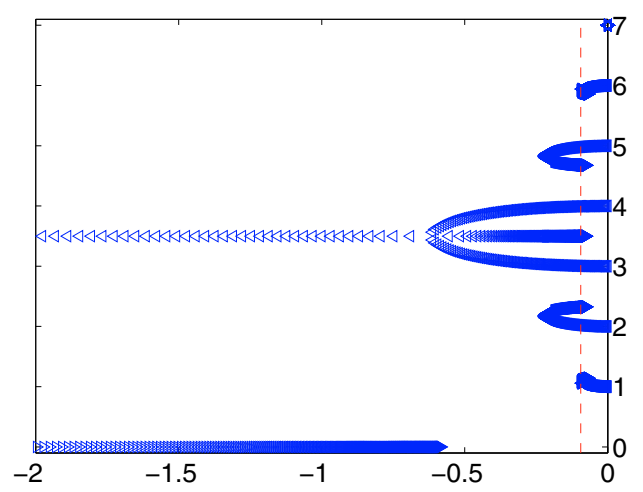

(F) $\quad a=3 \pi / 7, \mu_{*}=-0.206, b_{*}=2.69$

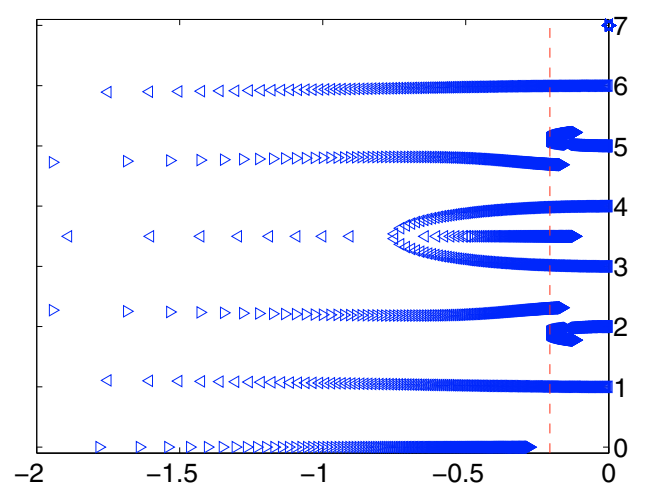

FiguRE 1. Eigen-trajectories as a function of touch, $b$, for ten choices of site, $a$.

and the quadratic has roots at $\left(-1 \pm \sqrt{b^{2}-3}\right) /(2-b)$, that coincide at $b=\sqrt{3}$. In terms of $\lambda$ this states that $\lambda=(3 /(2 \pi)) \ln (2-\sqrt{3})+3 i / 2$ is a double eigenvalue of $A(\pi / 3, \sqrt{3})$. It follows that $b=\sqrt{3}$ is in fact the correct touch when $a=\pi / 3$.

We now consider, for general $a=p \pi / q$, the nature of incoming, splitting and outgoing branches along the real axis and the line $\Im(\lambda)=q / 2$. 
(G) $a=\pi / 6, \mu_{*}=-0.117, b_{*}=2.2$

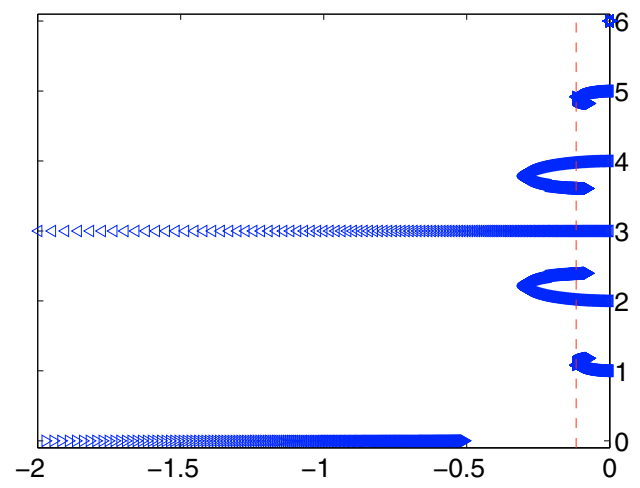

(I) $\mathrm{a}=\pi / 5, \mu_{\star}=-0.156, \mathrm{~b}_{*}=1.92$

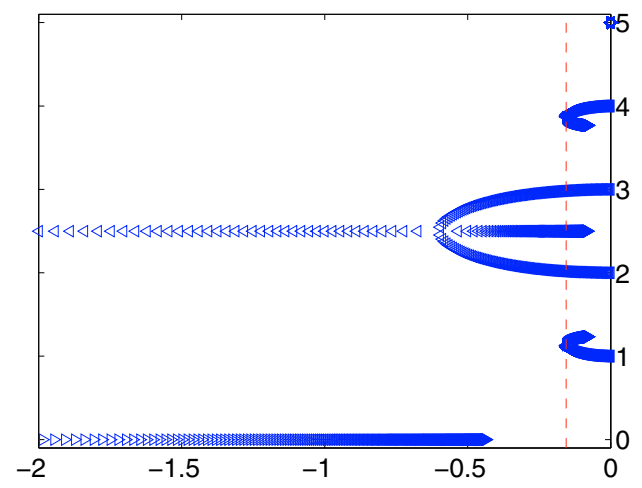

(H) $a=\pi / 3, \mu_{*}=-0.627, b_{*}=1.73$

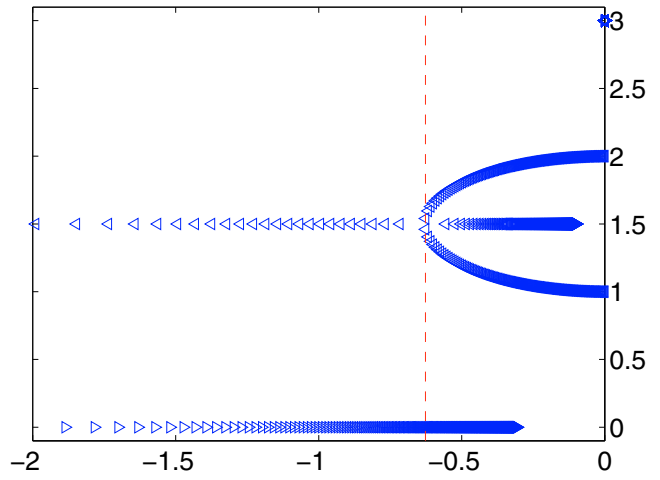

(J) $a=2 \pi / 5, \mu_{*}=-0.281, b_{*}=2.11$

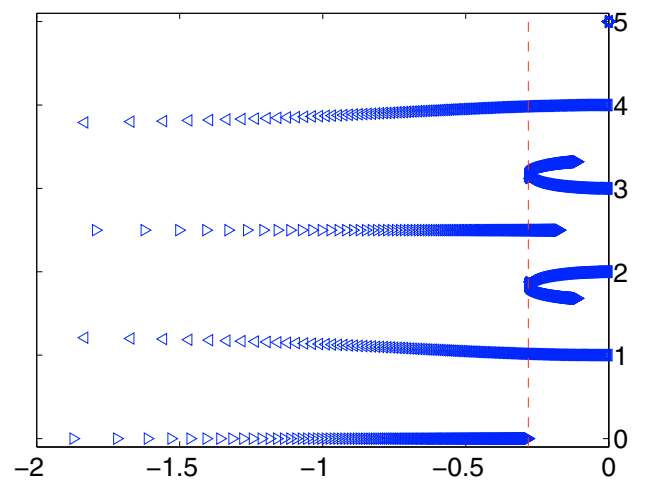

Figure 1. Continued.

Proposition 2.1. We suppose $a=p \pi / q$ and $\lambda=\alpha+i \beta$.

(0) If $b<2$ there exist no real eigenvalues. If $b>2$ then there exists precisely one real eigenvalue $\alpha_{0}(b)$. It is strictly increasing with $b$ and obeys

$$
\lim _{b \downarrow 2} \alpha_{0}(b)=-\infty \quad \text { and } \quad \lim _{b \rightarrow \infty} \alpha_{0}(b)=0 .
$$

(1) If $q$ is even and $b<2$ then $\alpha_{1}(b)+i q / 2$ is an eigenvalue and $\alpha_{1}(b)$ is strictly decreasing with $b$ and obeys

$$
\lim _{b \uparrow 2} \alpha_{1}(b)=-\infty .
$$

(2) If $q$ is odd and $p$ is even and $b>2$ then $\alpha_{2}(b)+i q / 2$ is an eigenvalue and $\alpha_{2}(b)$ is strictly increasing with $b$ and obeys

$$
\lim _{b \downarrow 2} \alpha_{2}(b)=-\infty \quad \text { and } \quad \lim _{b \rightarrow \infty} \alpha_{2}(b)=0 .
$$

(3) If $q$ is odd and $p$ is odd then there exists a unique $b^{*}<2$ and $\alpha\left(b^{*}\right)$ such that a double eigenvalue occurs at $\alpha\left(b^{*}\right)+i q / 2$. As $b$ exceeds $b^{*}$ this eigenvalue splits into the pair $\alpha_{ \pm}(b)+i q / 2$ satisfying

$$
\alpha_{-}(b)<\alpha\left(b^{*}\right)<\alpha_{+}(b), \quad \lim _{b \uparrow 2} \alpha_{-}(b)=-\infty \quad \text { and } \quad \lim _{b \rightarrow \infty} \alpha_{+}(b)=0 .
$$


Proof.

(0) If $\lambda=\alpha$ is real then (2.1) reads $b=-\sinh (\alpha \pi) /(\sinh (a \alpha) \sinh ((\pi-a) \alpha))$. As this is strictly increasing in $\alpha$ and takes values between 2 and $\infty$ the claim follows.

(1) If $q=2 n$ then $S(\alpha+i n ; p /(2 n), b)=(-1)^{n}\{\sinh (\pi \alpha)+b \cosh (a \alpha) \cosh ((\pi-a) \alpha)\}$ and so $b=-\sinh (\alpha \pi) /$ $(\cosh (a \alpha) \cosh ((\pi-a) \alpha))$. As this is strictly decreasing in $\alpha$ and takes values between 0 and 2 the claim follows.

(2) At $p=2 m, q=2 n+1, S(\alpha+i q / 2 ; a, b)=i(-1)^{n}\{\cosh (\pi \alpha)+b \cosh ((\pi-a) \alpha) \sinh (a \alpha)\}$ and so $b=-\cosh (\alpha \pi) /(\sinh (a \alpha) \cosh ((\pi-a) \alpha))$. As this is strictly increasing and takes values between 2 and $\infty$ the claim follows.

(3) At $p=2 m-1, q=2 n+1, S(\alpha+i q / 2 ; a, b)=i(-1)^{n}\{\cosh (\pi \alpha)+b \cosh (a \alpha) \sinh ((\pi-a) \alpha)\}$ and so

$$
F_{a}(\alpha) \equiv \frac{\cosh (a \alpha) \sinh ((\pi-a) \alpha)}{\cosh (\pi \alpha)}=-\frac{1}{b} .
$$

It is a simple matter to confirm

$$
F_{a}(0)=0, \quad-1 \leq F_{a}(\alpha) \leq 0, \quad \lim _{\alpha \rightarrow-\infty} F_{a}(\alpha) \rightarrow-1 / 2
$$

and that $F_{a}$ has a unique critical point, $\alpha_{a}$, satisfying $\alpha_{a}<-1 / 2$. As this critical point is the global minimizer it follows that (2.5) has (i) no solution for $b<b_{a} \equiv-1 / F_{a}\left(\alpha_{a}\right)$, (ii) one double root when $b=b_{a}$, (iii) exactly two roots when $b_{a}<b<2$ (with one going to $-\infty$ as $b \rightarrow 2$, and (iv) exactly one root when $b>2$.

We now show that this is the only possible multiple root.

Proposition 2.2. If $\lambda$ is a root of $S(\cdot ; a, b)$ then (i) its algebraic multiplicity can not exceed 2 and (ii) if it is double then $\Im \lambda=q / 2 \bmod q$.

Proof. We note that $S(\lambda ; a, b)=\sinh (\pi \lambda)+(b / 2) \cosh (\pi \lambda)-(b / 2) \cosh ((\pi-2 a) \lambda)$ and so

$$
\begin{aligned}
S^{\prime \prime}(\lambda ; a, b) & =\pi^{2} \sinh (\pi \lambda)+\pi^{2}(b / 2) \cosh (\pi \lambda)-(\pi-2 a)^{2}(b / 2) \cosh ((\pi-2 a) \lambda) \\
& =\pi^{2} S(\lambda ; a, b)-b a(2 a-\pi) \cosh ((\pi-2 a) \lambda)
\end{aligned}
$$

and so if $S(\lambda ; a, b)=S^{\prime \prime}(\lambda ; a, b)=0$ then $\cosh ((\pi-2 a) \lambda)=0$. The latter requires that $\lambda=i(n+1 / 2) \pi /(\pi-2 a)$ but $S(\lambda) \neq 0$.

For the second part we note that $\lambda=-(q /(2 \pi)) \log (z)$ where $z$ is a zero of $P$. We now show that every double root of $P$ is real. If $P(z)=P^{\prime}(z)=0$ then $q P(z)-z P^{\prime}(z)=0$. Dividing the latter by $b q$ and rearranging brings

while $z^{1-q} P^{\prime}(z)=0$ brings

$$
t z^{q-p}+(1-t) z^{p}=(b+2) / b, \quad t=p / q
$$

$$
t / z^{q-p}+(1-t) / z^{p}=(b-2) / b .
$$

If $z_{1} \equiv z^{q-p}$ and $z_{2} \equiv z^{p}$ then $(2.6)$ and (2.7) require

$$
t \Im\left(z_{1}\right)+(1-t) \Im\left(z_{2}\right)=0 \text { and } \quad t \frac{\Im\left(z_{1}\right)}{\left|z_{1}\right|^{2}}+(1-t) \frac{\Im\left(z_{2}\right)}{\left|z_{2}\right|^{2}}=0
$$

and so either $z_{1}$ and $z_{2}$ are real or $\left|z_{1}\right|=\left|z_{2}\right|$. The latter is ruled out by the fact that $|z|>1$. As $z^{q-p}$ and $z^{p}$ are both real and $q-p$ and $p$ are coprime it follows that $z$ is real. This gives the desired result, since $z=\exp (-2 \lambda \pi / q)$.

We now give a proposition which can be viewed as a "separation" result for the different branches of the spectrum. 
Proposition 2.3. If $a=p \pi / q$ and $b>0$ and $b \neq 2$ then the spectrum does not hit the horizontal lines $\lambda=\alpha+i \beta$ where

$$
\begin{aligned}
& \beta=\frac{k}{2}, \quad k=1, \ldots, 2 q-1, k \neq q \\
& \beta=k \frac{q}{2 p}, \quad k=1, \ldots, 2 p-1, k \neq p \\
& \beta=k \frac{q}{2(q-p)}, \quad k=1, \ldots, 2(q-p)-1, k \neq q-p .
\end{aligned}
$$

Proof. We claim that if $z$ is a root of (2.3) such that either $z^{q}$ or $z^{p}$ or $z^{q-p}$ is real, then necessarily $z$ is real. This will give the result since $z$ real means that the corresponding eigenvalue $\lambda$ satisfies $\Im \lambda=0$ or $\Im \lambda=q / 2$ or $\Im \lambda=q$ while the values of $\Im \lambda$ appearing in (2.8) correspond to $z^{q}$ or $z^{p}$ or $z^{q-p}$ real (and $z$ not real) and are therefore forbidden. Now, if $z^{p}$ is real then

$$
P(z ; b)=z^{q}\left\{(2-b)+\frac{b}{z^{p}}\right\}+b z^{p}-(b+2)=0
$$

immediately implies that $z^{q}$ is real. As $p$ and $q$ are coprime it follows that $z$ real.

Similarly, if $z^{q-p}$ is real then

$$
P(z ; b)=(2-b) z^{q-p} z^{p}+b z^{p}+b z^{q-p}-(2+b)=0
$$

implies that $z^{p}$ is real and so $z$ is real.

Next, if $z^{q}=t$ is real and $z^{p}=|z|^{p} \mathrm{e}^{i \theta}$ then

$$
P(z ; b)=b|z|^{p} \mathrm{e}^{i \theta}+\frac{b t}{|z|^{p}} \mathrm{e}^{-i \theta}+(2-b) t-(b+2)=0
$$

implies that $|z|^{p} \mathrm{e}^{i \theta}+t|z|^{-p} \mathrm{e}^{-i \theta}$ is real. Now $|z|>1$ implies that $\theta=0$, i.e., $z^{p}$ real, and so $z$ itself is real.

Recalling Figure 1 we set $r=\max (p, q-p)$ and distinguish the exit trajectories (where the magnitude of the real part is an unbounded increasing function of $0<b<2$ ) and return trajectories (where the magnitude of the real part is an unbounded decreasing function of $2<b<\infty)$ from the finite loops (bounded trajectory). Note that $q-r=\min (p, q-p)$ and that $q-2 r<0$.

For the behavior of the branches as $b \rightarrow+\infty$, we easily see (for example by writing the polynomial equation $\left.\left(1-z^{r}\right)\left(1-z^{q-r}\right)=2\left(1-z^{q}\right) / b\right)$, that the limit of all the branches are the points on the imaginary axis given by

$$
\begin{aligned}
& \lambda=i m \frac{q}{r}, \quad m=0, \ldots, r-1 \\
& \lambda=i m \frac{q}{q-r}, \quad m=0, \ldots, q-r-1 .
\end{aligned}
$$

We will see below that the first values are the end points of the finite loops, while the other ones are the end points of return trajectories.

Now, at $b=2$ we have only $r$ roots so $q-r$ roots must escape as $b \uparrow 2$. To follow these we write

$$
P(z ; b)=z^{r}\left\{(2-b) z^{q-r}+b+b z^{q-2 r}-(b+2) z^{-r}\right\}=0
$$

and deduce when $|z| \rightarrow+\infty$ that $z^{q-r} \sim \frac{b}{b-2}$. When $b \uparrow 2$, it follows that

$$
z \sim\left(\frac{b}{2-b}\right)^{1 /(q-r)} \exp (i \pi(1+2 m) /(q-r)), \quad m=0,1, \ldots, q-r-1 .
$$


Therefore, the possible imaginary parts of the exit trajectories $(b \uparrow 2)$ are

$$
\beta=\frac{q}{2(q-r)}(1+2 m), \quad m=0,1, \ldots, q-r-1 .
$$

Similarly, the return trajectories $(b \downarrow 2)$ emanate from points with imaginary part

$$
\beta=m \frac{q}{q-r}, \quad m=0,1, \ldots, q-r-1 .
$$

We now combine (2.9) with Proposition 2.3 to locate the starting points of the exit trajectories. Indeed, the first statement of (2.8) shows that there exists horizontal lines that the spectrum cannot cross every $i / 2$. Therefore, the only possible starting point for the trajectory that exits at $i \beta$, where $\beta=q(1+2 m) /(2(q-r))$, is $i \operatorname{round}(\beta)$ where round returns the closest integer. This is well defined except when $\beta$ is exactly half of an odd integer. Since $q-r$ is coprime with $q$, this can only happen if $q-r$ divides $2 m+1$. But, since $2 m+1<2(q-r)$, the only possible case is $2 m+1=q-r$. In this case, Proposition 2.1(3) instructs us that the branch is exactly the half-line $\beta=q / 2$ and it comes from both $(q+1) i / 2$ and $(q-1) i / 2$. Without loss we identify the lower point, $(q-1) i / 2$, with starting point of the loop that ends at $i q / 2$ after splitting, and identify the upper point, $(q+1) i / 2$, as the starting point of the trajectory that exits the complex plane at $\beta=q / 2$ after splitting. We have now proven:

Proposition 2.4. We set $r \equiv \max \{p, q-p\}$ and denote by round $^{ \pm}$the extension of round for which $\operatorname{round}^{-}(1 / 2)=0$ and round $^{+}(1 / 2)=1$.

(1) There exist $r-1$ proper loops, $\ell_{m}(b), 0 \leq b \leq \infty$,

$$
\ell_{m}(\infty)=i m q / r \quad \text { and } \quad \ell_{m}(0)=i \operatorname{round}^{-}(m q / r), \quad m=1, \ldots, r-1 .
$$

These are smooth where they avoid the multiple eigenvalue.

(2) There exist $q-r$ exit trajectories, $\varepsilon_{j}(b), 0 \leq b \leq 2$,

$$
\Im \varepsilon_{j}\left(2^{-}\right)=\frac{q(1+2 j)}{2(q-r)} \quad \text { and } \quad \varepsilon_{j}(0)=i \text { round }^{+}\left(\frac{q(1+2 j)}{2(q-r)}\right), \quad j=0, \ldots, q-r-1 .
$$

These are smooth where they avoid the multiple eigenvalue.

(3) There exist $q-r$ smooth return trajectories, $\rho_{j}(b), 2 \leq b \leq \infty$,

$$
\Im \rho_{j}\left(2^{+}\right)=\frac{j q}{q-r} \quad \text { and } \quad \rho_{j}(0)=i \text { round }\left(\frac{j q}{q-r}\right), \quad j=0, \ldots, q-r-1 .
$$

It follows that the nonharmonic spectral abscissa, $b \mapsto \mu(p \pi / q, b)$ of (1.5), is continuous and bounded below on $[0, \infty]$ and $\mu(p \pi / q, \infty)=0$. Hence, there exists a correct touch, i.e.:

Corollary 2.1. For integer coprime $p$ and $q$ the function $b \mapsto \mu(p \pi / q, b)$ attains its minimum over $[0, \infty]$ at a finite value $b_{*}(p, q)$.

Perusal of the root trajectories in Figure 1 suggests that $b_{*}$ occurs at a turning point (vertical tangent) of the smallest loop or perhaps along the fastest return trajectory. We now proceed to bound these loops.

\section{BOUNDS ON $\mu$ AND $b_{*}$}

We apply the determinant methods of Schur and Cohn to bound the moduli of the roots of

$$
R(w ; b)=P(w ; b) /(w-1)=(2+b) \sum_{j=0}^{p-1} w^{j}+2 \sum_{j=p}^{q-p-1} w^{j}+(2-b) \sum_{j=q-p}^{q-1} w^{j} .
$$


The principal tool is the Schur-Cohn Criterion.

Proposition 3.1 (Marden [23], Th. 43,1). The number of roots of $w \mapsto R(w ; b)$ in the ball of radius $c$ is the number of sign changes in a sequence of $q$ determinants,

$$
1, \Delta_{1}(b, c), \Delta_{2}(b, c), \ldots, \Delta_{q-1}(b, c) \quad \text { where } \quad \Delta_{k}(b, c)=\operatorname{det}\left(\begin{array}{cc}
L_{k} & U_{k} \\
U_{k}^{T} & L_{k}^{T}
\end{array}\right)
$$

and $L_{k}\left(U_{k}\right)$ is the $k$-by-k lower (upper) triangular Toeplitz matrix composed from the lowest (highest) $k$ coefficients of $w \mapsto R(c w ; b)$.

Starting at $k=1$ we find $L_{1}=2+b$ and $U_{1}=(2-b) c^{q-1}$ and so

$$
\Delta_{1}(b, c)=(2+b)^{2}-(2-b)^{2} c^{2 q-2} .
$$

In order to simplify the analysis of the higher determinants we shall assume, throughout the remainder of this section, that $p=1$. In that case

$$
L_{2}=\left(\begin{array}{cc}
2+b & 0 \\
2 c & 2+b
\end{array}\right) \quad \text { and } \quad U_{2}=\left(\begin{array}{cc}
(2-b) c^{q-1} & 2 c^{q-2} \\
0 & (2-b) c^{q-1}
\end{array}\right)
$$

and so

$$
\Delta_{2}(b, c)=(b-2)^{4} c^{4 q-4}-4(b-2)^{2} c^{2 q}+2 b^{2}\left(4-b^{2}\right) c^{2 q-2}-4(b+2)^{2} c^{2 q-4}+(b+2)^{4} .
$$

Now the turning points in $S$ correspond to turning points in $R$ which correspond to a double zero of one of the $b \mapsto \Delta_{k}(b, c)$. We detect such a zero by evaluating the associated discriminants. We denote by $D_{j}(c)$ the discriminant of $b \mapsto \Delta_{j}(b, c)$ and record $D_{1}(c)=64 c^{2 q-2}$ and

$$
\begin{gathered}
D_{2}(c)=2^{20} c^{6 q}\left(c^{q-1}+1\right)^{4}\left(c^{q-1}-1\right)^{4}\left(c^{q-4}+8 c^{2 q-4}+18 c^{q-2}+8+c^{q}\right) \\
\times\left(c^{q-4}-8 c^{2 q-4}+18 c^{q-2}-8+c^{q}\right) .
\end{gathered}
$$

We note $D_{1}$ never vanishes and that only the last factor in $D_{2}$ may vanish for $c>1$. Taking $q=4$ we find that $b \mapsto \Delta_{2}(b, c)$ has a double root when $c$ is a root of $7 c^{4}-18 c^{2}+7$. This has a unique root larger than 1 , namely

$$
c_{4}=(1+2 \sqrt{2}) / \sqrt{7}
$$

Transforming this back to the $\lambda$ variables we predict a turn in one of the eigenvalues at $\Re \lambda=-(2 / \pi) \log \left(c_{4}\right) \approx$ -0.2352 in agreement with Figure 1. To find the associated $b$ we must find the double root of $b \mapsto \Delta_{2}\left(b, c_{4}\right)$. This occurs at

$$
b_{4}=(6 / 5) \sqrt{2} \text {. }
$$

In order to determine whether this is the correct touch we must check the size of the remaining root of $R$. Writing $R\left(w ; b_{4}\right)=\left(2-b_{4}\right)\left(w-w_{1}\right)\left(w-w_{2}\right)\left(w-\bar{w}_{2}\right)$ we find $R\left(0 ; b_{4}\right)=\left(b_{4}-2\right) w_{1}\left|w_{2}\right|^{2}$ or

$$
w_{1}=\frac{2+b_{4}}{\left(b_{4}-2\right) c_{4}^{2}}=-3-2 \sqrt{2} .
$$

As this exceeds $c_{4}$ in magnitude, $b_{4}$ is indeed the correct touch. In the general case, a similar analysis allows us to get an easy lower bound for the spectral abscissa:

Proposition 3.2. Let $a=\pi / q, q \geq 4$ and $\alpha=(89+15 \sqrt{17}) / 64 \simeq 2.357$, then the spectral abscissa $\mu(a, b)$ satisfies

$$
\mu(a, b) \geq-\frac{q}{2 \pi(q-2)} \log \alpha .
$$


Proof. As mentioned above, a turning point may arrive for a value of $c$ such that

$$
E(c) \equiv-8 c^{2 q-4}+c^{q}+18 c^{q-2}+c^{q-4}-8=0 .
$$

Following the rule of signs of Descartes [23], Theorem 41.3, we know that the number $n$ of positive zeros and the number $m$ of change of signs in the coefficient of $E$ satisfy $n \leq m$ and $m-n$ even. Since $m=2, E(0)<0$, $E(1)>0$ and $E(+\infty)<0$, it follows that $E$ has two positive zeros and only one of them being larger than 1 . More precisely, since $E(2)$ can be written $E(2)=-8 * 2^{2 q-4}+\frac{89}{4} 2^{q-2}-8$ and $8 * 2^{q-2}>\frac{89}{4}$ for $q \geq 4$, we see that the unique root of $E$, say $c_{q}$, larger than 1 is smaller than 2 . We use this fact to improve the bound on $c_{q}$. Since

we get

$$
c_{q}^{2}+18+1 / c_{q}^{2}<2^{2}+18+1 / 2^{2}=89 / 4
$$

$$
0=E\left(c_{q}\right)<-8 c_{q}^{2 q-4}+\frac{89}{4} c_{q}^{q-2}-8
$$

and the bound $c_{q}^{q-2}<\alpha$ follows immediately. As the entire loop stays inside the disk of radius $c_{q}$ the bound (3.1) stems from the link between the modulus of $w$ and $\Re(\lambda)$.

It is also possible to get a lower bound for the spectral abscissa in terms of $b$. For that purpose, let us observe that looking at $\Delta_{1}(b, c)$, we immediately obtain that it becomes negative when $c$ crosses the value $((2+b) /(2-b))^{1 /(q-1)}$, therefore the polynomial $R$ has at least one root in the disk $|w|<((2+b) /(2-b))^{1 /(q-1)}$. Of course, if we now consider the next determinant $\Delta_{2}(b, c)$, we can improve this upper bound.

We factor $\Delta_{2}(b, c)$ into

$$
\begin{aligned}
\Delta_{2}(b, c)= & \left((b-2)^{2} c^{2(q-1)}+2(b-2) c^{q}+2(b+2) c^{q-2}-(b+2)^{2}\right) \\
& \times\left((b-2)^{2} c^{2(q-1)}-2(b-2) c^{q}-2(b+2) c^{q-2}-(b+2)^{2}\right) \equiv Q_{1}(b, c) Q_{2}(b, c)
\end{aligned}
$$

and look for the first zero of either $Q_{1}$ or $Q_{2}$. We observe that $Q_{2}(b, c) \leq Q_{1}(b, c)$ while $(b-2) c^{q}+(b+2) c^{q-2} \geq 0$, i.e.

Now, since

$$
Q_{2}(b, c) \leq Q_{1}(b, c) \Longleftrightarrow b \geq 2 \quad \text { or } \quad\left\{b<2 \text { and } 1 \leq c \leq\left(\frac{2+b}{2-b}\right)^{1 / 2}\right\} \text {. }
$$

$$
Q_{1}(b, 1)<0 \text { and } Q_{1}\left(b,\left(\frac{2+b}{2-b}\right)^{1 / 2}\right)=(b+2)^{2}\left(\left(\frac{2+b}{2-b}\right)^{q-3}-1\right)>0
$$

the first zero of $Q_{1}$ (and therefore of $P$ ) is less than $((2+b) /(2-b))^{1 / 2}$ and, therefore, we can assume that we are in the case $Q_{2}(b, c) \leq Q_{1}(b, c)$. At last, since $Q_{1}(b, 1)=-4 b<0$ and $Q_{2}(b, 1)=-12 b<0, Q_{1}(b, c)$ will vanish before $Q_{2}(b, c)$, so we can restrict ourselves to the study of $Q_{1}(b, c)$.

We begin with the case $b \geq 2$. Let $z_{0}$ be the positive root of

$$
R_{2}(z) \equiv(b-2)^{2} z^{2}+[2(b-2)+2(b+2)] z-(b+2)^{2} .
$$

Namely, $z_{0}=\left(-2 b+\sqrt{b^{4}-4 b^{2}+16}\right) /(b-2)^{2}$ for $b \neq 2$, and $z_{0}=2$ for $b=2$. We note that $z_{0}>1$, set $c_{0} \equiv z_{0}^{1 /(q-2)}>1$ and observe that if $b \geq 2$ then

$$
\begin{aligned}
Q_{1}\left(b, c_{0}\right) & =(b-2)^{2} c_{0}^{2} z_{0}^{2}+2(b-2) c_{0}^{2} z_{0}+2(b+2) z_{0}-(b+2)^{2} \\
& \geq(b-2)^{2} z_{0}^{2}+2(b-2) z_{0}+2(b+2) z_{0}-(b+2)^{2}=0 .
\end{aligned}
$$

Therefore, $c_{0}$ is an upper bound for the first zero of $P$ when $b \geq 2$. 


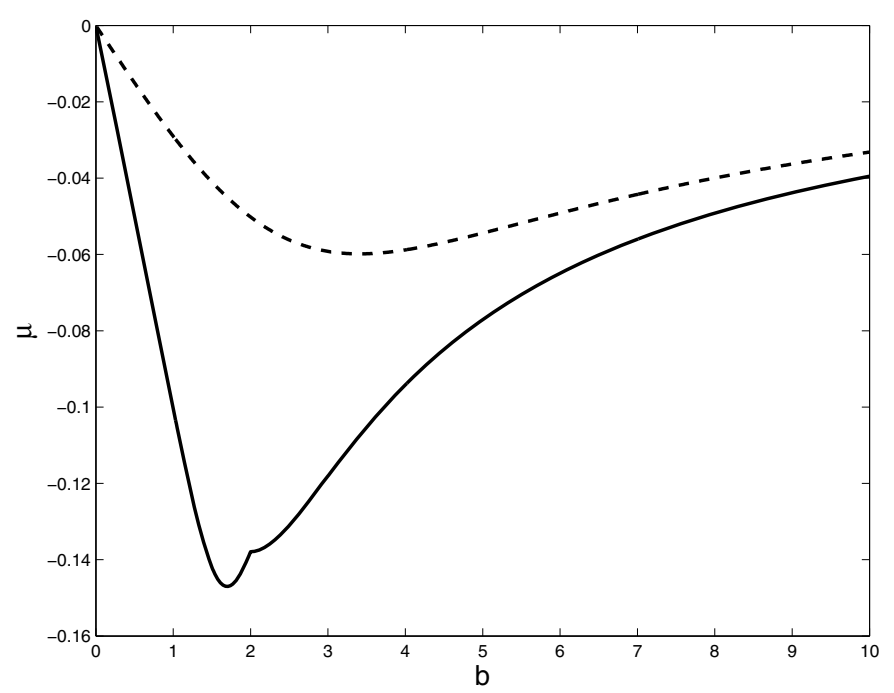

Figure 2. Estimate from above (solid line) for the spectral abscissa (dashed line) in the case $a=\pi / 10$.

We use the same kind of method in the case $b<2$. We introduce $z_{1}$ the (unique) positive root of

$$
R_{3}(z) \equiv(b-2)^{2} z^{3}+2(b-2) z^{2}+2(b+2) z-(b+2)^{2}=0 .
$$

Since $R_{3}(1)=-4 b<0$ and $R_{3}(2 /(2-b))=b^{2}(b+2) /(2-b)>0$ we see that $1<z_{1}<2 /(2-b)$. We set $c_{1} \equiv z_{1}^{1 /(q-2)}$ and observe that if $b \leq 2$ then

$$
\begin{aligned}
Q_{1}\left(b, c_{1}\right) & =(b-2)^{2} c_{1}^{2} z_{1}^{2}+2(b-2) c_{1}^{2} z_{1}+2(b+2) z_{1}-(b+2)^{2} \\
& =c_{1}^{2} z_{1}(b-2)\left[(b-2) z_{1}+2\right]+2(b+2) z_{1}-(b+2)^{2} \\
& \geq z_{1}^{2}(b-2)\left[(b-2) z_{1}+2\right]+2(b+2) z_{1}-(b+2)^{2}=0
\end{aligned}
$$

where we used the fact that $(b-2) z_{1}+2>0$ and $c_{1}^{2} \leq z_{1}$ (for $q \geq 4$ ). Therefore, $c_{1}$ is an upper bound for the first zero of $P$ when $b<2$. Now, using the link between the spectral abscissa $\mu$ and the moduli of the roots of $R$ we finally obtain the following result:

Proposition 3.3. Let $z_{1}(b)$ be the (unique) positive root of

$$
R_{3}(z) \equiv(b-2)^{2} z^{3}+2(b-2) z^{2}+2(b+2) z-(b+2)^{2}
$$

and $z_{0}(b)$ be the positive root of $R_{2}(z) \equiv(b-2)^{2} z^{2}+4 b z-(b+2)^{2}$. Then, for $q \geq 4$

$$
\mu(\pi / q, b) \geq-\frac{q}{2 \pi(q-2)} \begin{cases}\log \left|z_{1}(b)\right| & \text { when } b<2 \\ \log \left|z_{0}(b)\right| & \text { when } b \geq 2\end{cases}
$$

The estimate is particularly good for large values of $b$ as shown in Figure 2 drawn in the case $q=10$.

In order to get bounds for "the optimal touch" $b_{*}$, we now need an upper bound for the spectral abscissa, at least for a particular choice of $b$. Since we observe numerically that $b_{*}$ is not so far from the value $q / 3$, we choose this value.

Proposition 3.4. If $q \geq 6$ then $\mu(p \pi / q, q / 3) \leq-3 /(8 \pi q)$. 
Proof. We outline the key steps but suppress the many routine calculations. We recall the $S$ of $(2.2)$ and set $\phi(\alpha, \beta) \equiv|S(\alpha+i \beta)|^{2}$. We begin by working with $\beta$ fixed in the range of possible values corresponding to the different branches of the spectrum. We denote by $\mathcal{B}$ this set of possible values for $\beta$. We will often need to separate the cases of the closed loops and branches returning from infinity.

Step 1. The function $\alpha \mapsto \phi_{\alpha}(\alpha, \beta)$ is non decreasing for $-1 /(\pi q) \leq \alpha \leq 0$ (we just need to compute the second derivative $\phi_{\alpha \alpha}(\alpha, \beta)$ which is clearly non negative).

Step 2. The inequalities $\phi_{\alpha}(0, \beta) \geq 0$ and $\phi_{\alpha}(-1 /(\pi q), \beta)+\phi_{\alpha}(0, \beta) \geq 0$ hold. Actually, it is the longer and more technical part of the proof. For that, we write

$$
\frac{1}{\pi}\left(\phi_{\alpha}\left(\frac{-1}{\pi q}, \beta\right)+\phi_{\alpha}(0, \beta)\right)=A+B_{1} \frac{p}{3} \cos \frac{2(q-p)}{q} \beta \pi+B_{2} \frac{q-p}{3} \cos \frac{2 p}{q} \beta \pi
$$

with

$$
\begin{aligned}
A & =\frac{q}{3}+\frac{q}{3} \cosh \frac{2}{q}-\left(1+\frac{q^{2}}{36}\right) \sinh \frac{2}{q}-\frac{q(q-2 p)}{36} \sinh \frac{2(q-2 p)}{q^{2}} \\
B_{1} & =-1+\frac{q}{6} \sinh \frac{2 p}{q^{2}}-\cosh \frac{2 p}{q^{2}} \\
B_{2} & =-1+\frac{q}{6} \sinh \frac{2(q-p)}{q^{2}}-\cosh \frac{2(q-p)}{q^{2}}
\end{aligned}
$$

and we get the result using classical inequalities satisfied by the functions cosh and sinh and precise study of the function of $\beta$ defined in (3.2).

Step 3. We deduce from steps 1 and 2 that $\left|\phi_{\alpha}(\alpha, \beta)\right| \leq \phi_{\alpha}(0, \beta)$ for $-1 /(\pi q) \leq \alpha \leq 0$ and so $\phi(0, \beta)-\phi(\alpha, \beta) \leq$ $\phi_{\alpha}(0, \beta)|\alpha|$ there and then, $\phi(\alpha, \beta)$ (and also $S$ ) cannot vanish while

$$
\phi(0, \beta)-\phi_{\alpha}(0, \beta)|\alpha|>0 \Longleftrightarrow|\alpha|<\frac{\phi(0, \beta)}{\phi_{\alpha}(0, \beta)} \equiv \psi(\beta) .
$$

As a consequence, the spectral abscissa $\mu$ satisfies $\mu \leq-\psi(\beta)$ for each $\beta \in \mathcal{B}$.

Step 4. We find a lower bound for

$$
\psi(\beta)=\frac{\sin ^{2} \beta \pi+\frac{q^{2}}{9} \sin ^{2} \frac{p}{q} \beta \pi \sin ^{2} \frac{(q-p)}{q} \beta \pi}{2 \pi\left(\frac{p}{3} \sin ^{2} \frac{q-p}{q} \beta \pi+\frac{q-p}{3} \sin ^{2} \frac{p}{q} \beta \pi\right)}
$$

when $\beta$ varies in the range of possible values. We now examine separately the case of a closed loop and the case of a branch coming back from infinity (since $b=q / 3 \geq 2$, we do not need to look at the case of a branch going to infinity). For sake of simplicity, we assume here that $r=q-p$ (i.e. $q \geq 2 p$ ). The proof would be exactly the same in the other case.

The case of a loop finishing at $i m q /(q-p)$ corresponds to values of $\beta$ belonging to the interval $\left(k, \frac{m q}{q-p}\right)$ with $k \equiv \operatorname{round}(m q /(q-p))$ and $m$ are connected by a relation like $m q-k(q-p)=m_{0}$. Now, we use that on such an interval $\sin ^{2}((\pi-a) \beta) \leq \sin ^{2}(a \beta)$ to get

$$
\psi(\beta) \geq \frac{3}{2 \pi q}\left(\frac{q^{2}}{9} \sin ^{2}((\pi-a) \beta)+\frac{\sin ^{2} \beta \pi}{\sin ^{2}(a \beta)}\right) .
$$


We split the interval $I=\left(k, \frac{m q}{q-p}\right)$ into $I=\left(k, k+\frac{1}{2} \frac{m_{0}}{q-p}\right) \cup\left(k+\frac{1}{2} \frac{m_{0}}{q-p}, k+\frac{m_{0}}{q-p}\right)=I_{1} \cup I_{2}$. On $I_{1}$, we have

$$
\psi(\beta) \geq \frac{3}{2 \pi q} \frac{q^{2}}{9} \sin ^{2} \frac{q-p}{q} \beta \pi \geq \frac{q}{6 \pi} \sin ^{2} \frac{q-p}{q}\left(k+\frac{1}{2} \frac{m_{0}}{q-p}\right) \pi=\frac{q}{6 \pi} \sin ^{2} \frac{m_{0} \pi}{2 q} .
$$

At last, since on $[0, \pi / 12], \sin ^{2} x \geq\left(\frac{12 x}{\pi} \sin \frac{\pi}{12}\right)^{2}$, we get

$$
\sin ^{2} \frac{m_{0} \pi}{2 q} \geq \sin ^{2} \frac{\pi}{2 q} \geq \frac{144}{\pi^{2}} \frac{\pi^{2}}{4 q^{2}} \sin ^{2} \frac{\pi}{12}=\frac{18}{q^{2}}\left(1-\frac{\sqrt{3}}{2}\right),
$$

therefore, on $I_{1}$

$$
\psi(\beta) \geq \frac{3(2-\sqrt{3})}{2 \pi q}
$$

On $I_{2}$, we write:

the right-hand side is equal to

$$
\psi(\beta) \geq \frac{3}{2 \pi q} \frac{\sin ^{2} \beta \pi}{\sin ^{2} \frac{p}{q} \beta \pi} \geq \frac{3}{2 \pi q} \frac{\sin ^{2}\left(k \pi+\frac{1}{2} \frac{m_{0} \pi}{q-p}\right)}{\sin ^{2} \frac{p \pi}{q}\left(k+\frac{m_{0}}{q-p}\right)},
$$

therefore, on $I_{2}$

$$
\frac{3}{2 \pi q} \frac{\sin ^{2} \frac{m_{0} \pi}{2(q-p)}}{\sin ^{2} \frac{m_{0} \pi}{(q-p)}}=\frac{3}{2 \pi q} \frac{1}{4 \cos ^{2} \frac{m_{0} \pi}{2(q-p)}}
$$

$$
\psi(\beta) \geq \frac{3}{8 \pi q} .
$$

In the case of a branch coming back from infinity, which corresponds to an interval like $\left(k, \frac{j q}{q-r}\right)$ with $k \equiv$ $\operatorname{round}(j q /(q-r))$ and $j q-k(q-r)=q-j_{0}$, we use the same idea and we get the same inequalities as (3.5) and (3.6).

As a consequence of the upper and lower bounds on the nonharmonic spectral abscissa we obtain the following bounds on the correct touch.

Proposition 3.5. Let us denote by $b_{*}(p, q)$ the correct touch, i.e., the minimizer of $b \mapsto \mu(p \pi / q, b)$. If $p=1$ and $q \geq 6$ then

$$
b_{1}(q) \leq b_{*}(p, q) \leq b_{2}(q)
$$

where

$$
\begin{aligned}
& b_{1}(q)=\frac{2 z^{3}-z^{2}-z+2+\sqrt{-7 z^{4}+18 z^{3}-7 z^{2}}}{z^{3}-1} \\
& b_{2}(q)=\frac{2\left(z^{2}-z+1\right)+2 \sqrt{2 z(2-z)\left(z-\frac{1}{2}\right)}}{z^{2}-1}
\end{aligned}
$$

and $z=\exp \left(3(q-2) / 4 q^{2}\right)$.

Proof. To find these bounds, it suffices to look at the intersection of the curve giving the lower bound with the line $\mu=-3 /(8 \pi q)$. Or, equivalently, to find $b$ such that (in the language of Prop. 3.3) $z_{0}(b)$ and $z_{1}(b)$ equal $\exp \left(3(q-2) / 4 q^{2}\right)$. We obtain the result by plugging this value of $z$ in the equation defining $z_{0}(b)$ and $z_{1}(b)$ respectively and solving in $b$. 


\section{Regarding the Root Vectors}

Recall that Rayleigh was already aware of the fact that pointwise damping destroys the "normal co-ordinates" or orthonormal basis of eigenmodes enjoyed by the undamped wave operator. For our purposes however it will suffice to show that the (generalized) eigenmodes, or root vectors, are isomorphic to an orthonormal base. In other words, we show that these modes comprise a Riesz basis for the energy space, $X$. We begin with a specification of these modes.

In addition to the harmonic modes in (1.2) we have the nonharmonic modes

$$
V_{k, n}(x)=y_{k, n}(x)\left[1 / \lambda_{k, n} 1\right], \quad y_{k, n}(x)= \begin{cases}\sinh \left(\lambda_{k, n}(\pi-a)\right) \sinh \left(\lambda_{k, n} x\right) & \text { if } x<a \\ \sinh \left(\lambda_{k, n} a\right) \sinh \left(\lambda_{k, n}(\pi-x)\right) & \text { if } x>a\end{cases}
$$

where $k=2, \ldots, q$ and $n$ runs through the integers.

If $\lambda_{k, n}=\lambda_{k+1, n}$ then, in addition to its eigenvector $V_{k, n}$, there exists the generalized eigenvector $V_{k+1, n}=[\tilde{y} \tilde{z}]$ for which $\left(A-\lambda_{k}\right) V_{k+1, n}=V_{k, n}$. In components this reads

$$
\tilde{z}=\lambda_{k, n} \tilde{y}+y_{k, n} / \lambda_{k, n} \quad \text { and } \quad \tilde{y}^{\prime \prime}-\left(\lambda_{k, n}+b \delta_{a}\right) \tilde{z}=y_{k}
$$

The first into the latter yields

$$
\tilde{y}^{\prime \prime}-\lambda_{k, n}^{2} \tilde{y}-\lambda_{k, n} b \delta_{a} \tilde{y}=\left(2+b \delta_{a} / \lambda_{k, n}\right) y_{k, n}
$$

that is, $\tilde{y}$ is the $H_{0}^{1}(0, \pi)$ function satisfying

$$
\tilde{y}^{\prime \prime}-\lambda_{k, n}^{2} \tilde{y}=2 \begin{cases}\sinh \left(\lambda_{k, n}(\pi-a)\right) \sinh \left(\lambda_{k, n} x\right) & \text { if } x<a \\ \sinh \left(\lambda_{k, n} a\right) \sinh \left(\lambda_{k, n}(\pi-x)\right) & \text { if } x>a\end{cases}
$$

and the transmission condition

$$
\tilde{y}^{\prime}\left(a^{+}\right)-\tilde{y}^{\prime}\left(a^{-}\right)=\lambda_{k, n} b \tilde{y}_{k, n}(a)+b y_{k, n}(a) / \lambda_{k, n} .
$$

The solution to (4.2)-(4.3) is, piecewise,

$$
\tilde{y}(x)=\frac{1}{\lambda_{k, n}}\left\{\begin{array}{l}
(\pi-a) \cosh \left(\lambda_{k, n}(\pi-a)\right) \sinh \left(\lambda_{k, n} x\right)+x \cosh \left(\lambda_{k, n} x\right) \sinh \left(\lambda_{k, n}(\pi-a)\right) \\
a \cosh \left(\lambda_{k, n} a\right) \sinh \left(\lambda_{k, n}(\pi-x)\right)+(\pi-x) \cosh \left(\lambda_{k, n}(\pi-x)\right) \sinh \left(\lambda_{k, n} a\right)
\end{array}\right.
$$

We now show that the root vectors comprise a Riesz Basis for $X$. In some related cases, e.g., Kergomard et al. [17], the underlying isomorphism can be constructed by hand. We follow here the indirect approach of Bari, e.g., Young [34], Theorem 1.9, and show that they comprise a complete Bessel sequence and are biorthogonal to a set that is also complete and Bessel. To begin, as $\left\{V_{1, n}\right\}_{n \neq 0}$ is an orthonormal sequence in $X$, Bessel's inequality reads

$$
\sum_{n= \pm 1}^{ \pm \infty}\left|\left\langle V_{1, n}, V\right\rangle\right|^{2} \leq\|V\|^{2}, \quad \forall V \in X
$$

and so we need only show:

Proposition 4.1. If $V \in X$ then $\sum_{n}\left|\left\langle V_{k, n}, V\right\rangle\right|^{2}<\infty$ for each $k=2, \ldots, q$. 
Proof. We write $V=[y z]$ and find

$$
\left\langle V_{k, n}, V\right\rangle=\left(1 / \lambda_{k, n}\right) \int_{0}^{\pi} y_{k, n}^{\prime} \bar{y}^{\prime} \mathrm{d} x+\int_{0}^{\pi} y_{k, n} \bar{z} \mathrm{~d} x .
$$

Now, as $\lambda_{k, n}=\lambda_{k, 0}-i q n$,

$$
\begin{aligned}
\frac{1}{\lambda_{k, n}} \int_{0}^{\pi} y_{k, n}^{\prime} \bar{y}^{\prime} \mathrm{d} x= & (-1)^{n(q-p)} \sinh \left(\lambda_{k, 0}(\pi-a)\right) \int_{0}^{a} \cosh \left(\left(\lambda_{k, 0}-i n q\right) x\right) \bar{y}^{\prime}(x) \mathrm{d} x \\
& -(-1)^{n p} \sinh \left(\lambda_{k, 0} a\right) \int_{a}^{\pi} \cosh \left(\left(\lambda_{k, 0}-i n q\right)(\pi-x)\right) \bar{y}^{\prime}(x) \mathrm{d} x
\end{aligned}
$$

As $\{n q\}_{n}$ is a separated sequence it follows from Young [34], Theorem 4.4, that $\mathrm{e}^{i n q}$ is a Bessel sequence in $L^{2}(-\pi, \pi)$ and hence the sequence (4.5) is square summable in $n$. This same argument allows us to conclude that the second term in (4.4) is also square summable in $n$. Finally, given our explicit representation of the root vectors associated with double eigenvalues, the argument above may be used to show that these too constitute a Bessel sequence.

These same estimates hold for the adjoint system

$$
\begin{aligned}
A^{*}(a, b) & =\left(\begin{array}{cc}
0 & -I \\
-\partial_{x x} & 0
\end{array}\right) \quad x \neq a \\
D\left(A^{*}(a, b)\right) & =\left\{[f, g] \in\left(H_{0}^{1}(0, \pi) \cap H^{2}(0, a) \cap H^{2}(a, \pi)\right) \times H_{0}^{1}(0, \pi): f^{\prime}\left(a^{+}\right)-f^{\prime}\left(a^{-}\right)=-b g(a)\right\}
\end{aligned}
$$

for its root vectors are $W_{k, n}(x)=\bar{y}_{k, n}(x)\left[1 / \bar{\lambda}_{k, n}-1\right]$. It is a simple matter to confirm that this sequence is biorthogonal to $\left\{V_{k, n}\right\}$.

Regarding completeness, as our pointwise damping is an unbounded 'perturbation' of the skew wave operator we may not argue as in Cox and Zuazua [9]. Instead, following Krein and Nudelman [20], Cox and Zuazua [10] and Ammari et al. [1], we invoke the simple trace criterion of Livšic.

Proposition 4.2 (Krein and Langer [19], Sect. 2.5). If $H$ is Hilbert and $T: H \rightarrow H$ is linear and compact and $T_{\Re} \equiv\left(T+T^{*}\right) / 2$ is nonpositive and of finite trace then

$$
\operatorname{tr}\left(\mathrm{T}_{\Re}\right) \leq \sum_{\nu_{\mathrm{n}} \in \sigma(\mathrm{T})} \Re \nu_{\mathrm{n}}
$$

where the $\nu_{n}$ are repeated according to their algebraic multiplicity. Equality holds in (4.6) if and only if the root vectors of $T$ are complete in $H$.

It is now a simple matter to confirm that equality holds in (4.6). We note that an alternate route to completeness has recently been established in the works of Guo and Xie [14] and Xu and Guo [33].

Proposition 4.3. $\operatorname{tr}\left(A^{-1}(a, b)_{\Re}\right)=-b(\pi-a) a / \pi$.

Proof. If $[f g]=A^{-1}(a, b)[u v]$ then $g=u$ and $f^{\prime \prime}=v+b \delta_{a} u$ with $f(0)=f(\pi)=0$. Hence

$$
f(x)=\int_{0}^{x}(x-t) v(t) \mathrm{d} t-(x / \pi) \int_{0}^{\pi}(\pi-t) v(t) \mathrm{d} t-(b / \pi) u(a) \psi(x)
$$

where

$$
\psi(x)= \begin{cases}(\pi-a) x & \text { if } x<a, \\ a(\pi-x) & \text { if } x>a .\end{cases}
$$


Similarly, if $[f g]=A^{-1}(a, b)^{*}[u v]$ then $g=-u$ and $f^{\prime \prime}=-v+b \delta_{a} u$ with $f(0)=f(\pi)=0$. Hence, as above

$$
f(x)=-\int_{0}^{x}(x-t) v(t) \mathrm{d} t+(x / \pi) \int_{0}^{\pi}(\pi-t) v(t) \mathrm{d} t-(b / \pi) u(a) \psi(x) .
$$

Combining these calculations we find $A^{-1}(a, b)_{\Re}[u v]=[-(b / \pi) u(a) \psi 0]$, and so the range of $A^{-1}(a, b)_{\Re}$ is spanned by the unit vector $e_{1} \equiv[\psi 0] / \sqrt{a \pi(\pi-a)}$. The claim follows from $\operatorname{tr} A^{-1}(a, b)_{\Re}=\left\langle A^{-1}(a, b)_{\Re} e_{1}, e_{1}\right\rangle$.

The polynomial representation of our shooting function affords the elementary factorization

$$
\begin{aligned}
S(\lambda ; p \pi / q, b) & =-\frac{1}{4} \exp (\lambda \pi) P(\exp (-2 \pi \lambda / q) ; b) \\
& =\frac{1}{4} \exp (\lambda \pi)(b-2) \prod_{k=1}^{q}\left(\exp (-2 \pi \lambda / q)-w_{k}\right) .
\end{aligned}
$$

The beauty of this representation is that sums of eigenvalues, as required by (4.6), appear on differentiation with respect to $\lambda$. The computation of these sums will be facilitated by

$$
\sum_{n} \frac{1}{(n+\alpha)^{2}+\beta^{2}}=\frac{\pi}{2 \beta} \frac{\sinh (2 \pi \beta)}{\cosh ^{2}(\pi \beta)-\cos ^{2}(\pi \alpha)}
$$

that follows by summing the residues of $-\pi \cot (\pi z) /\left((z+\alpha)^{2}+\beta\right)$.

Proposition 4.4. If $b \geq 0$ and $b \neq 2$ then the shooting function satisfies

$$
S(\lambda ; p \pi / q, b)=\pi \lambda-\pi \lambda^{2} \sum_{k=1}^{q} \sum_{n} \Re\left(1 / \lambda_{k, n}\right)+O\left(\lambda^{3}\right) .
$$

Proof. We simply differentiate (4.7) with respect to $\lambda$ and make frequent use of the fact that $w_{1}=1$. To begin, with $a=p \pi / q$,

$$
S_{\lambda}(\lambda ; a, b)=\frac{\pi}{4} \mathrm{e}^{\lambda \pi}(b-2) \prod_{k=1}^{q}\left(\mathrm{e}^{-2 \pi \lambda / q}-w_{k}\right)-\frac{\pi}{2 q} \mathrm{e}^{\lambda \pi(1-2 / q)}(b-2) \sum_{j=1}^{q} \prod_{k \neq j}\left(\mathrm{e}^{-2 \pi \lambda / q}-w_{k}\right) .
$$

The first term vanishes at $\lambda=0$ and the products in the second term each vanish except when $j=1$. Hence

$$
S_{\lambda}(0 ; a, b)=\frac{\pi}{2 q}(2-b) \prod_{k=2}^{q}\left(1-w_{k}\right)=\left.\frac{\pi}{2 q} \frac{P(w)}{w-1}\right|_{w=1}=\pi
$$

as claimed. Proceeding to the second derivative

$$
\begin{aligned}
S_{\lambda \lambda}(\lambda ; a, b)= & \frac{\pi^{2}}{4} \mathrm{e}^{\lambda \pi}(b-2) \prod_{k=1}^{q}\left(\mathrm{e}^{-2 \pi \lambda / q}-w_{k}\right) \\
& -\frac{\pi^{2}(q-1)}{q^{2}} \mathrm{e}^{\lambda \pi(1-2 / q)}(b-2) \sum_{j=1}^{q} \prod_{k \neq j}\left(\mathrm{e}^{-2 \pi \lambda / q}-w_{k}\right) \\
& +\frac{\pi^{2}}{q^{2}} \mathrm{e}^{\lambda \pi(1-4 / q)}(b-2) \sum_{j=1}^{q} \sum_{i=1}^{q} \prod_{k \notin\{i, j\}}\left(\mathrm{e}^{-2 \pi \lambda / q}-w_{k}\right) .
\end{aligned}
$$


At $\lambda=0$ this becomes

$$
\begin{aligned}
S_{\lambda \lambda}(0 ; a, b) & =\frac{\pi^{2}(q-1)}{q^{2}}(2-b) \prod_{k=2}^{q}\left(1-w_{k}\right)+\frac{2 \pi^{2}}{q^{2}}(b-2) \sum_{j=2}^{q} \prod_{k \notin\{1, j\}}\left(1-w_{k}\right) \\
& =\frac{2 \pi^{2}(q-1)}{q}-\frac{4 \pi^{2}}{q} \sum_{k=2}^{q} \frac{1}{1-w_{k}} \\
& =\frac{2 \pi^{2}}{q} \sum_{k=2}^{q}\left(\frac{1-w_{k}}{1-w_{k}}-\frac{2}{1-w_{k}}\right) \\
& =\frac{2 \pi^{2}}{q} \sum_{k=2}^{q} \frac{w_{k}+1}{w_{k}-1}
\end{aligned}
$$

and so it remains only to link the $w_{k}$ back to their $\lambda_{k, n}$. Well, it follows directly from (2.4) that

$$
\Re\left(1 / \lambda_{k, n}\right)=\frac{-\log \left|w_{k}\right|}{2 \pi q} \frac{1}{\log ^{2}\left(\left|w_{k}\right|\right) /\left(4 \pi^{2}\right)+\left(n+\theta_{k} /(2 \pi)\right)^{2}} .
$$

With $\alpha=\theta_{k} /(2 \pi)$ and $\beta=\log \left(\left|w_{k}\right|\right) /(2 \pi)$ it now follows from (4.8) that

$$
\begin{aligned}
\sum_{n} \Re\left(1 / \lambda_{k, n}\right) & =\frac{-\log \left|w_{k}\right|}{2 \pi q} \frac{\pi^{2}}{\log \left|w_{k}\right|} \frac{\sinh \left(\log \left|w_{k}\right|\right)}{\cosh ^{2}\left(\log \left(\left|w_{k}\right|\right) / 2\right)-\cos ^{2}\left(\theta_{k} / 2\right)} \\
& =\frac{-\pi}{4 q} \frac{\left|w_{k}\right|-\left|w_{k}\right|^{-1}}{\left(\left|w_{k}\right|+2+\left|w_{k}\right|^{-1}\right) / 4-\cos ^{2}\left(\theta_{k} / 2\right)} \\
& =\frac{\pi}{q} \frac{1-\left|w_{k}\right|^{2}}{\left|w_{k}\right|^{2}-2 \Re w_{k}+1} .
\end{aligned}
$$

In order to reconcile (4.10) and (4.9) we note that if $w_{k}$ is real then

$$
\frac{1-\left|w_{k}\right|^{2}}{\left|w_{k}\right|^{2}-2 \Re w_{k}+1}=\frac{w_{k}+1}{1-w_{k}}
$$

while if $w_{k}$ is not real then

$$
\frac{1-\left|w_{k}\right|^{2}}{\left|w_{k}\right|^{2}-2 \Re w_{k}+1}+\frac{1-\left|\bar{w}_{k}\right|^{2}}{\left|\bar{w}_{k}\right|^{2}-2 \Re \bar{w}_{k}+1}=\frac{w_{k}+1}{1-w_{k}}+\frac{\bar{w}_{k}+1}{1-\bar{w}_{k}} .
$$

As each $w_{k}$ is either real or occurs with its complex conjugate, we have shown,

$$
S_{\lambda \lambda}(0 ; a, b)=-2 \pi \sum_{k=1}^{q} \sum_{n} \Re\left(1 / \lambda_{k, n}\right)
$$

as claimed.

Comparing now the MacLaurin developments of $S$ as represented in (2.1) and the above proposition, we find

$$
\pi \lambda+b(\pi-a) a \lambda^{2}+O\left(\lambda^{3}\right)=\pi \lambda-\pi \lambda^{2} \sum_{\lambda_{n} \in \sigma(a, b)} \Re\left(1 / \lambda_{n}\right)+O\left(\lambda^{3}\right)
$$




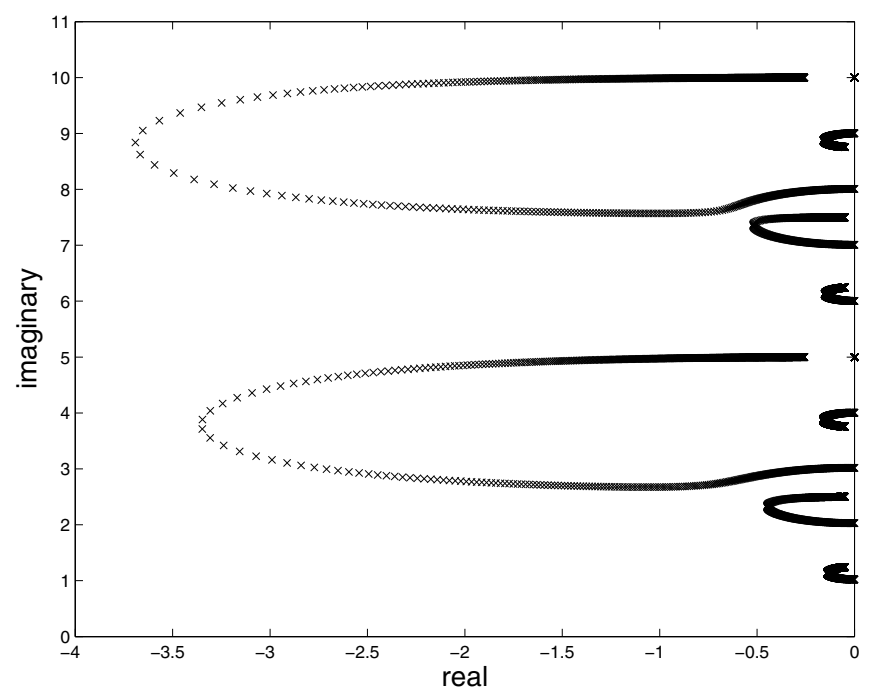

Figure 3. A few root paths of $(5.3)$ when $p / q=1 / 5, k=0.2$, and $b$ runs from 0 to 8 .

and so, recalling Proposition 4.2, we have shown that, when $b \neq 2$,

$$
\sum_{\lambda_{k, n} \in \sigma(a, b)} \Re\left(1 / \lambda_{k, n}\right)=\operatorname{tr}\left(\left(A^{-1}\right)_{\Re}\right) .
$$

It follows that the root vectors are complete in $X$ and hence comprise a Riesz basis in $X$, and, more to the point, that the set of nonharmonic root vectors $\left\{V_{k, n}: k=2, \ldots, q ; n \in \mathbf{Z}\right\}$ is a Riesz basis for $\mathcal{H}^{\perp}$. From here it is easy to see, e.g., [9], that the nonharmonic spectral abscissa, (1.5), indeed coincides with the decay rate on $\mathcal{H}^{\perp}$.

\section{Dimensions And EXtensions}

Though it has been convenient to work in the setting of Bamberger et al. it remains to see whether the 'correct' touch is in fact practical. To see this we start from the dimensional problem

$$
T u_{x x}-\rho u_{t t}+\gamma \delta(x-p \ell / q) u_{t}=0, \quad 0<x<\ell .
$$

In terms of the new space and time variables, $y=\pi x / \ell$ and $\tau=(\pi t / \ell) \sqrt{T / \rho}$ we recover our initial equation

$$
u_{y y}-u_{\tau \tau}+b \delta(y-p \pi / q) u_{\tau}=0, \quad 0<y<\pi \quad \text { where } \quad b=\frac{\gamma}{\sqrt{T \rho}} .
$$

We note that Cuzzucoli and Lombardo [11] consider finger damping up to $8 \sqrt{T \rho}$. On an absolute scale, if we take, e.g., the string studied in Cox [8], where $T=27 \mathrm{~m} \cdot \mathrm{kg} \cdot \mathrm{s}^{-2}$ and $\rho=0.0015 \mathrm{~kg} \cdot \mathrm{m}^{-1}$ we find that $\sqrt{T \rho} \approx 0.2 \mathrm{~kg} \cdot \mathrm{s}^{-1}$ corresponds to light damping.

Finally, following, e.g., Birch and Srinivasan [5], we note that the interaction of string and finger is much more complex than our 1-parameter pointwise damper. From the 15-parameter model of [5] we may abstract an effective fingertip stiffness, $k>0$, and so consider

$$
u_{t t}-u_{x x}+\delta_{a}\left(b u_{t}+k u\right)=0 .
$$


This breaks the lovely symmetry of the shooting function,

$$
S(\lambda ; a, b)=\lambda \sinh (\lambda \pi)+(b \lambda+k) \sinh (\lambda a) \sinh (\lambda(\pi-a)) .
$$

and so also destroys the simple structure of the spectrum. In comparing Figures 1 and 3 we see at once both the unfolding of the multiple eigenvalue and the genesis of the exit and return trajectories. Although the $q i$ periodicity is broken, it appears that the full spectral abscissa is attained on one of the first $q$ trajectories.

It remains of course to determine whether the correct touch in the sense of Bamberger, Rauch and Taylor is indeed the most pleasing to the ear. If in fact the nonharmonic spectral abscissa is the right measure to minimize then Figure 1 seems to indicate a number of improvements over current practice. More precisely, modern practice, as dictated by Zukovsky [36], has the musician lightly finger at $\ell / q$ and yet, for odd $q>3$ Figure 1 states that fingering at $2 \ell / q$ is better, i.e., $\mu\left(2 \ell / q, b_{*}(2, q)\right)<\mu\left(\ell / q, b_{*}(1, q)\right)$. By this reasoning it appears that touching simultaneously at $\ell / q$ and $2 \ell / q$ should in turn further enhance the effect.

\section{REFERENCES}

[1] K. Ammari, A. Henrot and M. Tucsnak, Asymptotic behavior of the solutions and optimal location of the actuator for the pointwise stabilization of a string. Asymptot. Anal. 28 (2001) 215-240.

[2] A. Bamberger, J. Rauch and M. Taylor, A model for harmonics on stringed instruments. Arch. Rational Mech. Anal. 79 (1982) 267-290.

[3] G. Banat, Masters of the Violin, Sonatas for the Violin, Jean-Joseph Cassanéa de Mondonville 5. Johnson Reprint (1982).

[4] D. Bernoulli, Réflexions et éclaircissemens sur les nouvelles vibrations des cordes exposées dans les mémoires de 1747 and 1748. Histoire de l'Academie royale des sciences et belles lettres $\mathbf{9}$ (1753) 148-172.

[5] A.S. Birch and M.A. Srinivasan, Experimental determination of the viscoelastic properties of the human fingerpad. Touch Lab Report 14, RLE TR-632, MIT, Cambridge (1999).

[6] J.T. Cannon and S. Dostrovsky, The Evolution of Dynamics, Vibration Theory from 1687 to 1742. Springer, New York (1981).

[7] T. Christensen, Rameau and Musical Thought in the Enlightenment. Cambridge (1993).

[8] S.J. Cox, Aye there's the rub, An inquiry into how a damped string comes to rest, in Six Themes on Variation, R. Hardt Ed., AMS (2004) 37-58.

[9] S. Cox and E. Zuazua, The rate at which energy decays in a damped string. Comm. Partial Diff. Eq. 19 (1994) $213-243$.

[10] S. Cox and E. Zuazua, The rate at which energy decays in a string damped at one end. Indiana U. Math. J. 44 (1995) 545-573.

[11] G. Cuzzucoli and V. Lombardo, A physical model of the classical guitar, including the player's touch. Comput. Music J. 23 (1999) 52-69.

[12] F.W. Galpin, Monsieur Prin and his trumpet marine. Music Lett. 14 (1933) 18-29.

[13] C. Girdlestone, Jean-Philippe Rameau. Cassell, London (1957).

[14] B.-Z. Guo and Y. Xie, A sufficient condition on Riesz basis with parenthesis of nonself-adjoint operator and application to a serially connected string system under joint feedbacks. SIAM J. Control Optim. 43 (2004) 1234-1252.

[15] H. Helmholtz, On the Sensations of Tone. Dover (1954).

[16] S. Jaffard, M. Tucsnak and E. Zuazua, Singular internal stabilization of the wave equation. J. Diff. Eq. 145 (1998) $184-215$.

[17] J. Kergomard, V. Debut and D. Matignon, Resonance modes in a 1-D medium with two purely resistive boundaries: calculation methdos, orthogogonality and completeness. J. Acoust. Soc. Am. 119 (2006) 1356-1367.

[18] I. Kovács, Zur Frage der Seilschwingungen und der Seildämpfung. Die Bautechnik 59 (1982) 325-332.

[19] M.G. Krein and H. Langer, On some mathematical principles in the linear theory of damped oscillations of continua I. Integr. Equ. Oper. Theory 1 (1978) 364-399.

[20] M.G. Krein and A.A. Nudelman, On direct and inverse problems for the boundary dissipation frequencies of a nonuniform string. Soviet Math. Dokl. 20 (1979) 838-841.

[21] S. Krenk, Vibrations of a taut cable with an external damper. J. Appl. Mech. 67 (2000) 772-776.

[22] K.S. Liu, Energy decay problems in the design of a pointwise stabilizer for string vibrating systems. SIAM J. Control Optim. 26 (1988) $1248-1256$

[23] M. Marden, Geometry of Polynomials. AMS (1966).

[24] D.C. Miller, Anecdotal History of the Science of Sound. Macmillan, New York (1935).

[25] J.-P. Rameau, Generation Harmonique, Facsimile of 1737 Paris Ed., Broude Brothers, New York (1966).

[26] J.W.S. Rayleigh, Theory of Sound, Vol. 1. Dover (1945).

[27] F. Roberts, A discourse concerning the musical notes of the trumpet, and trumpet-marine, and of the defects of the same. Philosophical Transactions 16 (1692) 559-563.

[28] J. Sauveur, Systéme général des intervalles des sons et son application à tous les systémes et à tous les instrumens de musique, Mémoires de l'Académie royale des sciences 1701. Amsterdam (1707) 390-482. 
[29] B. Taylor, De Moti Nervi Tensi. Philosophical Transactions 28 (1713) 26-32.

[30] C. Truesdell, The Rational Mechanics of Flexible or Elastic Bodies, 1638-1788, introduction to Leonhardi Euleri Opera Omnia Vols. 10 and 11, Series 2, Leipzig (1912).

[31] J. Tyndall, Sound. D. Appleton (1875).

[32] J. Wallis, Concerning a new musical discovery. Philosophical Transactions 12 (1677) 839-842.

[33] G.-Q. Xu and B.-Z. Guo, Riesz basis property of evolution equations in Hilbert spaces and application to a coupled string equation. SIAM J. Control Optim. 42 (2003) 966-984.

[34] R.M. Young, An Introduction to Nonharmonic Fourier Series. Academic Press, San Diego (2001).

[35] T. Young, A Course of Lectures on Natural Philosophy and the Mechanical Arts. Johnson Reprint (1971).

[36] P. Zukovsky, On violin harmonics. Perspectives of New Music 6 (1968) 174-181. 